\title{
Detección de Staphylococcus pseudintermedius resistentes a meticilina y a otros antimicrobianos de uso habitual en la clínica en piodermias caninas
}

\section{Detection of Staphylococcus pseudintermedius resistant to methicillin and to other antimicrobials commonly used in canine pyodermias}

\author{
Giacoboni $\mathbf{G I}^{1^{*}, 2}$, Vinocur $\mathrm{F}^{2}$, Fauret $\mathrm{N}^{2}$, Grandinetti $\mathrm{J}^{3}$, Manzuc $\mathrm{P}^{4}$ \\ ${ }^{1}$ Departamento de Microbiología, ${ }^{2}$ Cátedra de Microbiología, Departamento de Microbiología, ${ }^{3}$ Cátedra de \\ Patología General Veterinaria, Departamento de Ciencias Preclínicas, ${ }^{4} \mathrm{H}$ ospital Escuela, Departamento de \\ Clínica. Facultad de Ciencias Veterinarias, Universidad Nacional de La Plata \\ *Correo electrónico del autor: giacoboni@fcv.unlp.edu.ar
}

\begin{abstract}
Resumen: Se estudiaron 96 muestras provenientes de caninos que presentaban diferentes formas clínicas de piodermia. El objetivo fue detectar, mediante pruebas fenotípicas, la presencia de Staphylococcus pseudintermedius $\mathrm{y}$, mediante antibiograma, la resistencia antimicrobiana a la meticilina y a otros antimicrobianos de uso común en la práctica clínica, ambos métodos utilizados en el laboratorio de diagnóstico veterinario. S. pseudintermedius se identificó en 91 animales $(94,8 \%$ y y la resistencia a la meticilina se detectó en 31 cepas (34\%). De ellas, 21 (93,5\%) expresaron multirresistencia a 4,5 y 6 antimicrobianos. El perfil de resistencia predominante fue OXA-ERY-CLIN-TMS-CIP-CMP. Entre los otros antibióticos probados, los que presentaron mayor porcentaje de resistencia fueron: trimetoprima sulfametoxazol $61 / 67 \%$, eritromicina $59 / 64,4 \%$, clindamicina $55 / 60 \%$ y ciprofloxacina $47 / 52 \%)$. Todas las cepas fueron sensibles a vancomicina, nitrofurantoína y rifampicina. La prueba $D$ (conocida como D-test) se utilizó para evaluar la resistencia inducible a la clindamicina. Se concluye que la identificación fenotípica de S. pseudintermedius, así como el antibiograma, son herramientas importantes para colaborar en la utilización responsable de los antimicrobianos en medicina veterinaria.
\end{abstract}

Palabras clave: identificación fenotípica, Staphylococcus pseudintermedius, antibiograma, meticilinorresistencia, piodermia canina

\begin{abstract}
Ninety-six samples from dogs with different clinical forms of pyodermia were studied, with the objective of detecting the presence of Staphylococcus pseudintermedius by phenotypic tests and the antimicrobial resistance to methicillin and other antimicrobials commonly used in clinical practice. S. pseudintermedius was identified in 91 animals (94.8\%) and methicillin resistance was detected in 31 strains (34\%). Of these, 21 (93.5\%) expressed multiresistance to 4, 5 and 6 antimicrobials. The predominant resistance profile was OXA-ERY-CLIN-TMS-CIP-CMP. Among other antibiotics tested, the ones with the highest percentage of resistance were: trimethoprim-sulfamethoxazole $(67 \%)$, erythromycin $(64.4 \%)$, clindamycin (60\%) and ciprofloxacin (52\%). All strains were sensitive to vancomycin, nitrofurantoin and rifampicin. The D-test was performed to evaluate inducible resistance to clindamycin. It is concluded that the phenotypic identification of $S$. pseudintermedius as well as the antibiogram are important tools to contribute to the responsible use of antimicrobials in veterinary medicine.
\end{abstract}

Key words: phenotypical identification, Staphylococcus pseudintermedius, antibiogram, methicillin resistance, canine pyoderma 


\section{Introducción}

La piodermia canina es una entidad a la que habitualmente se enfrenta el médico veterinario en la práctica clínica. Las opciones del tratamiento varían, según la profundidad y localización de la lesión, desde la aplicación de antisépticos y antimicrobianos locales hasta el uso de antibióticos sistémicos.

La clasificación del agente etiológico más frecuentemente involucrado cambió luego de la revisión de la clasificación de los estafilococos coagulasa positivos. Así, la descripción de la nueva especie «Staphylococcus pseudintermedius Devriese et al., 2005» completó la agrupación con varios estafilococos estrechamente relacionados en el grupo llamado Staphylococcus intermedius (GSI), que hoy involucra a $S$. intermedius, S. pseudintermedius y S. delphini grupo A y B. Como consecuencia de la revisión de esta clasificación, se asume que la mayoría de los estafilococos aislados de caninos e identificados como S. intermedius son S. pseudintermedius. Sin embargo, la determinación de especie mediante pruebas bioquímicas presenta limitaciones, ya que existen escasas pruebas bioquímicas/metabólicas que los diferencien (Sasaki et al., 2007).

Esta bacteria forma parte de la microbiota normal del perro; constituye, en la boca $57 \%$ de la microbiota, en el periné $52 \%$ y en ingles $23 \%$. Es un patógeno oportunista y no causa enfermedad, a menos que la inmunocompetencia del hospedador se encuentre disminuida o que la barrera cutánea se altere por factores como dermatitis atópica, procedimientos médicos, quirúrgicos o factores ambientales (Bannoehr \& Guardabassi, 2012).

Desde la descripción de S. pseudintermedius se conoce su sensibilidad a los antibióticos betalactámicos, pero a partir de 2006 surgió la resistencia a la meticilina (S. pseudintermedius resistente a la meticilina, SPRM). Se trata de una situación similar a la de $S$. aureus resistente a la meticilina (SARM) por la presencia del gen mec A (Weese \& van Duijkeren, 2010), a pesar de que en S. pseudintermedius se describen actualmente otros genes mec (Chanchaithong et al., 2016).

La prueba de sensibilidad a los antimicrobianos por difusión (antibiograma), utilizada comúnmente en los laboratorios de diagnóstico es, en la práctica, la que puede ayudar al veterinario clínico a seleccionar el antibiótico adecuado para instaurar un tratamiento criterioso y con mayor probabilidad de éxito. El antibiograma se realiza según pautas y recomendaciones de instituciones internacionales, tales como el European Committee on Antimicrobial Susceptibility Testing (EUCAST) y el Clinical and Laboratory Standars Institute (CLSI). Este último es el que se toma como referencia en nuestro país para la interpretación de las pruebas de sensibilidad.
La detección de resistencia a meticilina y a otros antibióticos betalactámicos mediada por el gen $\operatorname{mec} A$ en S. pseudintermedius ha sido motivo de controversia a través del tiempo.

En 2009, los grupos de investigadores encabezados por Schissler y por Bemis evaluaron la utilidad de los puntos de corte del CLSI para detectar resistencia a meticilina en aislamientos de S. pseudintermedius de caninos. Demostraron que el disco de cefoxitina usado para predecir resistencia a meticilina en $S$. aureus y estafilococos coagulasa negativos no es útil para S. pseudintermedius, pero sí lo es el disco de oxacilina. Las normas Vet01-S28, de 2013 del CLSI, incluyeron puntos de corte para oxacilina a $S$. pseudintermedius, dado que es el adecuado para detectar la meticilinorresistencia en esta especie bacteriana.

EI CLSI tiene puntos de corte establecidos para $S$. aureus aislados de humanos con el disco de cefoxitina $30 \mu \mathrm{g}(\mathrm{S} \geq 22 \mathrm{~mm} / \mathrm{R} \leq 21 \mathrm{~mm})$. En 2013, este instituto eliminó de las tablas de estafilococos a la oxacilina, dado que la cefoxitina es mejor marcador de resistencia a meticilina mediada por el gen $\operatorname{mec} A$. En 2016 incorporó puntos de corte de oxacilina para S. pseudintermedius ( $\mathrm{S} \geq 18 \mathrm{~mm} / \mathrm{R} \leq 17 \mathrm{~mm}$ ) y consignó que ni el disco de cefoxitina ni la concentración inhibitoria mínima (CIM) de cefoxitina son apropiados para detectar resistencia a meticilina mediada por el gen mec $A$ en esta especie (CLSI, 2016).

Para el laboratorio de diagnóstico bacteriológico, la evolución en la clasificación de los estafilococos, así como las normas para la interpretación de su comportamiento frente a los antimicrobianos in vitro son motivos de actualización y desafío, pues la correcta identificación de la especie de estafilococo es indispensable para predecir la resistencia antimicrobiana.

A pesar de que los avances tecnológicos para identificar S. pseudintermedius, como las técnicas moleculares (Bannoehr et al., 2009) y de espectrometría de masa (Descristóphoris et al., 2011), permiten una identificación certera, la mayoría de los laboratorios de diagnóstico veterinario utilizan la identificación fenotípica.

Este trabajo recopila la identificación de $S$. pseudintermedius aislados de perros con diagnóstico clínico de piodermia en el transcurso de 10 meses, así como la resistencia que presentaron a la meticilina y a otros antimicrobianos de prescripción habitual, por medio de la utilización de pruebas fenotípicas y de antibiograma, respectivamente. Se pretende alertar a los médicos veterinarios clínicos sobre la importancia que tienen el laboratorio de diagnóstico veterinario en la identificación del microorganismo y las pruebas de sensibilidad antimicrobiana como herramientas de suma utilidad para la elección y prescripción correcta del fármaco, así como sobre su uso prudente 
para prevenir o evitar la extensión de la resistencia antimicrobiana.

\section{Materiales y métodos}

Se obtuvieron y enviaron al laboratorio muestras de 96 caninos de entre 6 meses y 14 años de edad, con diagnóstico clínico de piodermia. Se registraron: edad, raza, sexo, datos de enfermedades de base, tipo de piodermia según su localización y evolución, antimicrobianos utilizados en tratamientos previos a la toma de muestras. Se procesaron 96 hisopados de piel. Las muestras se tomaron de tres lesiones diferentes por hisopo, en el período comprendido entre febrero y diciembre de 2016, de caninos provenientes de la Ciudad Autónoma de Buenos Aires, Gran Buenos Aires y La Plata, República Argentina.

En el laboratorio, se sembraron en caldo salado ( $\mathrm{NaCl}$ al 6,5\%) para su preenriquecimiento selectivo a $37^{\circ} \mathrm{C}$ durante $24 \mathrm{~h}$ y, posteriormente, en agar manitol salado. Las colonias sospechosas fueron repicadas en agar tripticasa soya para obtener un cultivo puro. La identificación de especies se realizó mediante las siguientes pruebas bioquímicas: coagulasa, Voges Proskauer, fermentación de trehalosa, manitol y maltosa, hidrólisis de la arginina y I-pirrolidonilarilamidasa.

La sensibilidad a los antimicrobianos se determinó mediante el método de difusión en agar según normas M2-A 11 del CLSI. Se utilizaron discos de oxacilina $1 \mu \mathrm{g}(\mathrm{OXA})$, eritromicina $15 \mu \mathrm{g}(\mathrm{ERY})$, clindamicina $2 \mu \mathrm{g}(\mathrm{CLIN})$, ciprofloxacina $5 \mu \mathrm{g}(\mathrm{CIP})$, nitrofurantoína $30 \mu \mathrm{g}(\mathrm{NIT})$, rifampicina $5 \mu \mathrm{g}$ (RIF), cloranfenicol $30 \mu \mathrm{g}$ (CMP), minociclina $30 \mu \mathrm{g}$ (MIN) trimetoprima-sulfametoxazol $25 \mu \mathrm{g}$ (TMS) y vancomicina $5 \mu \mathrm{g}$ (VAN) (Laboratorio Britania S. A, CABA, Argentina). Los resultados del método de difusión con oxacilina, eritromicina, clindamicina, cloranfenicol y trimetoprima sulfametoxazol se interpretaron según las normas CLSI (2013) para aislamientos de animales Vet01-S28, mientras que, con el resto de los antimicrobianos, los resultados se interpretaron según las normas CLSI M100-S26 (2016) para aislamientos de humanos, por no disponer de puntos de corte para aislamientos de animales. Se evaluó el fenotipo de resistencia a macrólidos utilizando la prueba D, según lo describen Steward et al. (2005) para $S$. aureus. La prueba D se realiza colocando los discos de eritromicina y clindamicina separados por una distancia de $15 \mathrm{~mm}$ entre sí, de borde a borde. El desarrollo en forma de letra $D$ en la zona circular de inhibición alrededor del disco de clindamicina indica la presencia de MLSB inducible.

\section{Resultados}

S. pseudintermedius se aisló e identificó en $n=91(94,8 \%)$ de los animales mediante identifica- ción fenotípica. Los antimicrobianos que presentaron mayores registros de resistencia fueron TMS $n=61$ (67\%), seguidos por ERY $n=59(64,8 \%)$ y CLIN $n=55$ $(60,4 \%)$. Hubo 15 aislamientos (16\%) que mostraron sensibilidad a todas las drogas probadas (Tabla 1).

La resistencia a la meticilina, detectada utilizando el disco de oxacilina según la recomendación del CLSI, se presentó en 31 cepas (34\%). De ellas, $21(93,5 \%)$ expresaron multirresistencia a 4,5 y 6 antimicrobianos. El perfil de resistencia predominante fue ERY-CLIN-TMS-CIP, seguido del perfil OXA-ERYCLIN-TMS-CIP-CMP (Tabla 2).

Tabla 1. Resistencia a los antimicrobianos probados en 91 cepas de S. pseudintermedius aisladas de caninos con piodermia.

\begin{tabular}{llc}
\hline Antimicrobiano & \multicolumn{2}{c}{$\begin{array}{c}\text { Cantidad de aislamien- } \\
\text { tos resistentes }\end{array}$} \\
\hline Oxacilina & 31 & $(34 \%)$ \\
Eritromicina & 59 & $(65 \%)$ \\
Clindamicina & 55 & $(60 \%)$ \\
Ciprofloxacina & 47 & $(52 \%)$ \\
Trimetoprima-sulfametoxazol & 61 & $(67 \%)$ \\
Cloranfenicol & 15 & $(16 \%)$ \\
Minociclina & 1 & $(1 \%)$ \\
Rifampicina & 0 & $(0 \%)$ \\
Nitrofurantoína & 0 & $(0 \%)$ \\
Vancomicina & 0 & $(0 \%)$ \\
\hline
\end{tabular}

Tabla 2. Fenotipo de resistencia.

\begin{tabular}{|c|c|c|}
\hline Resistencia & Fenotipo de resistencia & $\mathrm{N}^{\circ}$ de cepas \\
\hline 7ATB ${ }^{a}$ & $\begin{array}{l}O X A^{b}-E R Y^{c}-C L I N^{d}-T_{M S}^{e}-C I P^{f}- \\
C M P^{g}-R I^{h}\end{array}$ & 3 \\
\hline 6 ATB & OXA-ERY-CLIN-TMS-CIP-CMP & 8 \\
\hline 6 ATB & OXA-ERY-CLIN-TMS-MINO'-CMP & 1 \\
\hline 5 ATB & ERY-CLIN-TMS-CIP-CMP & 1 \\
\hline 5 ATB & OXA-ERY-CLIN-TMS-CIP & 9 \\
\hline 4 ATB & ERY-CLIN-TMS-CIP & 15 \\
\hline 4 ATB & OXA-ERY-CLIN-TMS & 4 \\
\hline 4 ATB & OXA-ERY-CLIN-CMP & 1 \\
\hline 4 ATB & OXA-ERY-CLIN-CIP & 3 \\
\hline 3 ATB & ERY-CLIN-TMS & 8 \\
\hline 3 ATB & ERY-TMS-CIP & 1 \\
\hline 3 ATB & ERY-CLIN-CIP & 1 \\
\hline 2 ATB & TMS-CIP & 3 \\
\hline 2 ATB & ERY-CLIN & 1 \\
\hline 2 ATB & CIP-CMP & 1 \\
\hline 1 ATB & TMS & 8 \\
\hline 1 ATB & ERY & 3 \\
\hline 1 ATB & CIP & 3 \\
\hline 1 ATB & OXA & 2 \\
\hline Ningún ATB & & 15 \\
\hline
\end{tabular}

aantimicrobiano, boxacilina, 'eritromicina, dclindamicina, etrimetoprima/sulfametoxazol, ${ }^{f}$ ciprofloxacina, ${ }^{g}$ Cloranfenicol, ${ }^{h}$ rifampicina, iminociclina 
La resistencia a macrólidos en estafilococos puede deberse al mecanismo de eflujo o a la modificación del sitio blanco macrólido-lincosamidaestreptogramina B (MLSB). La resistencia MLSB puede ser constitutiva o inducible tras la exposición a un macrólido.

De los 59 aislamientos resistentes a ERY, 4 $(6,8 \%)$ presentaron sensibililidad a CLIN y prueba $\mathrm{D}$ negativas, correspondiente al fenotipo $\mathrm{M}$ de eflujo. Los 55 aislamientos restantes presentaron resistencia a ERY y CLI correspondiente al fenotipo MLSB constitutivo.

Hubo solo un aislamiento resistente a minociclina (1\%). Todos los aislamientos fueron sensibles a VAN, RIF y a NIT.

\section{Discusión y conclusiones}

La colonización con S. pseudintermedius en perros es común, ya que la bacteria reside en su piel y mucosas. Se informó su aislamiento hasta en un $90 \%$ de los animales portadores sanos (Griffeth et al., 2008). La piodermia asociada con este patógeno oportunista es una infección común de los caninos que frecuentemente requiere de tratamientos prolongados. Sin embargo, el tratamiento se ha visto dificultado por el aumento de la resistencia a los antimicrobianos (Bannoehr \& Guardabassi, 2012). La infección surge, generalmente, cuando las barreras cutáneo-mucosas son alteradas, ya sea por lesiones ocasionadas por procedimientos quirúrgicos o medicamentosos, por dermatitis parasitarias, dermatitis atópicas o enfermedades metabólicas (Bannoehr \& Guardabassi, 2012). En cuanto a las formas de presentación de las dermatitis, las formas superficial y generalizada fueron las más frecuentes, coincidiendo con la bibliografía (Bloom et al., 2014).

La infección causada por SPRM es un desafío emergente por la aparición de cepas multirresistentes a betalactámicos, macrólidos, lincosamidas, aminoglucósidos sulfonamidas y cloranfenicol y las limitadas opciones terapéuticas que presentan algunos casos. Ante este problema, surgieron investigaciones como la de Waleed Younis et al. (2017) para hallar compuestos con otros sitios de acción como, por ejemplo, compuestos de la enzima 3-hidroxi-3-metilglutarilcoenzima, que aún están en evaluación.

Se registró un $34 \%$ de resistencia a la meticilina con el disco de oxacilina e interpretándolo con los puntos de corte de CLSI para aislamientos de S. pseudintermedius en animales (Vet01-S28 CLSI, 2013), resistencia que generalmente está determinada por la presencia del gen mec $A$ (Weese \& van Duijkeren, 2010). Algunos investigadores discrepan en la interpretación de esos datos, argumentando que el rol del gen mec $A$ con el informe extendido de resistencia a los compuestos betalactámicos se estableció sobre la base clínica y microbiológica de $S$. aureus en humanos, y que fue trasladado a S. pseudintermedius en medicina veterinaria sin estudios específicos en animales. Mientras se aguardan estas investigaciones, se recomienda informar los aislamientos que presentan resistencia a la oxacilina como resistentes a los antibióticos betalactámicos que se utilizan en medicina veterinaria, con una evaluación clínica del caso antes de cambiar el tratamiento si, por ejemplo, los animales están siendo tratados con amoxicilina-clavulánico o cefalexina (Guardabassi et al., 2017).

La observación de altas resistencias a TMS, CIP y ERY se registraron en otro estudio similar de piodermias caninas en la Universidad de Santa María, Brasil, pero con valores más bajos que los nuestros (TMS 29,9\%, ERY 17,7\%, CIP 18,8 \%). En el mismo estudio detectaron $0,6 \%$ de resistencia a la vancomicina, resistencia que no se encontró entre los 91 aislamientos de este trabajo (da Silva et al., 2014).

La resistencia a macrólidos en estafilococos puede deberse al mecanismo de eflujo o a la modificación del sitio blanco macrólido-lincosamidaestreptogramina B (MLSB). La resistencia MLSB puede ser constitutiva o inducible tras la exposición a un macrólido. Cuando se observa resistencia a la eritromicina y la clindamicina tiene halo de sensibilidad pero presenta achatamiento, la prueba $D$ es positiva y significa que hay resistencia inducible. Según CLSI, los aislamientos que presenten prueba $\mathrm{D}$ positiva, a pesar de presentar halo de sensibilidad a clindamicina, se deben informar resistentes, ya que podrían desarrollar resistencia intra-tratamiento y llevar al fracaso terapéutico.

En nuestro trabajo no encontramos resistencia inducible a clindamicina (MLSB inducible), como tampoco la informaron Faires et al. (2009) en un estudio de SPRM en perros y gatos, ni Vigo et al. (2015) en un estudio retrospectivo de $S$. pseudintermedius de diferentes casos clínicos en caninos. Gold \& Lawhon (2013), aunque hallaron solamente $1,32 \%$ de resistencia inducible (8/608), enfatizaron la importancia de la prueba $\mathrm{D}$, debido a que la alternativa recomendable ante una falla terapeútica con amoxicilina-clavulánico y cefalosporinas de primera generación es la clindamicina, debido a su eficacia, seguridad y buena distribución en piel. Sin embargo, los valores de resistencia para clindamicina hallados $(60 \%)$ fueron altos y similares a los encontrados por Moodley et al. (2014) en la revisión que realizaron entre 1980-2013 con un rango de porcentajes entre 60-80.

Las interpretaciones de los resultados de los antibiogramas podrían ser objetadas en los casos en los que no están establecidos los puntos de corte para 
dicho antimicrobiano en medicina veterinaria. Tal es el caso de la minociclina y la doxiciclina que fueron evaluadas por Hnot et al. (2015). Estos autores observaron que muchas cepas portadoras de genes tetM, que confieren resistencia a tetraciclina por metilación ribosomal, eran sensibles a ambos antibióticos si se interpretaban con los puntos de corte para aislamientos de humanos, es decir que estos puntos de corte no son apropiados para animales.

La definición y la clasificación de las especies en bacteriología han ido cambiando y la taxonomía tiende a diversificarse debido a la proliferación de técnicas moleculares que se registran día a día y forman parte de bases de datos actualizadas.

En esta especie bacteriana se describieron dos casettes cromosómicos ( $\mathrm{SCCmec}$ ) nuevos que portan mec A: SCCmec II-III y SCCmec VII. Este último es un nuevo elemento propio de $S$. pseudintermedius que no se encuentra en $S$. aureus (Descloux et al., 2008). Se completó la secuenciación de los 3 tipos clonales dominantes de S. pseudintermedius meticilinorresistentes (ST 84, ST 71 y ST 68), todos con el gen mec A (Riley et al., 2016).

A pesar de estos avances, en la práctica clínica y en los laboratorios veterinarios se siguen utilizando, tanto para la identificación bacteriana como para determinar la sensibilidad a los antimicrobianos, pruebas bioquímicas convencionales y antibiograma por difusión.

En el caso de la resistencia a la meticilina mediada por el gen mec $A$, la prueba de oro para confirmarla es la PCR, pero las normas CLSI 2013 ya establecieron luego de las apreciaciones realizadas por Schissler et al. (2009) y Bemis et al. (2009) que el disco de oxacilina puede ser, como para el caso de $S$. aureus el disco de cefoxitina, el predictor de dicha resistencia. Las pruebas que evalúan características macroscópicas, reacciones bioquímicas y metabólicas así como el antibiograma por difusión son las que pueden ofrecerse a los médicos veterinarios para la resolución de los casos de dermatitis por $S$. pseudintermedius, más aún cuando aparecen cepas multirresistentes. Sin embargo, son las que pueden ofrecerse a los médicos veterinarios para la resolución de los casos de dermatitis por $S$. pseudintermedius, más aun cuando aparecen cepas multirresistentes.

Este estudio de laboratorio permitió conocer la prevalencia de S. pseudintermedius, a partir de muestras de piel, en pacientes caninos con piodermias en un período de 10 meses en el año 2016. El estudio del comportamiento de la bacteria frente a los diferentes antimicrobianos utilizados corrientemente en la práctica clínica fue de valor para que el médico veterinario tomara decisiones para la elección del tratamiento.
Estas acciones colaboran con el uso prudente de los antimicrobianos en medicina veterinaria, tal como lo propone la Organización Mundial de Sanidad Animal (OIE), para preservar la eficacia de estos valiosos medicamentos, considerados un bien público mundial, en los animales, el hombre y el medio ambiente.

\section{Conflicto de intereses}

Todos los autores declaran que no existe conflicto de intereses, incluyendo las relaciones financieras, personales o de otro tipo con otras personas u organizaciones que pudieran influir de manera inapropiada en el trabajo.

\section{Bibliografía}

Bannoehr J, Franco A, lurescia M, Battisti A, Fitzgerald JR. 2009. Molecular diagnostic identification of Staphylococcus pseudintermedius. Journal of Clinical Microbiology. 47:46971. doi: 10.1128/JCM.01915-08.

Bannoehr J, Guardabassi L. 2012. Staphylococcus pseudintermedius in the dog: taxonomy, diagnostics, ecology, epidemiology and pathogenicity. Veterinary Dermatology. 23(4):253-66, e51-2. doi: 10.1111/j.1365-3164.2012.01046.x.

Bemis DA, Jones RD, Frank LA, Kania SA. 2009. Evaluation of susceptibility test breakpoints used to predict mec Amediated resistance in Staphylococcus isolated from dogs. Journal of Veterinary Diagnostic Investigation. 21:53-84. doi. org/10.1177/104063870902100108.

Bloom P. 2014. Canine superficial bacterial folliculitis: current understanding of this etiology, diagnosis and treatment. Veterinary Journal. 199:217-22. doi.org/10.1016/j. tvjl.2013.11.014.

Chanchaithong P, Prapasarakul N, Perreten V,Schwendenera S. 2016. Characterization of a novel composite Staphylococcal cassette chromosome mec in methicillin-resistant Staphylococcus pseudintermedius from Thailand. Antimicrobial Agents and Chemotherapy. 60(2):1153-7. doi: 10.1128/ AAC.02268-15.

Clinical Laboratory Standards Institute (CLSI). 2012. M2-A11: Performance Standards for Antimicrobial Disk Susceptibility Test: Approved Standard- Eleventh Edition. Clinical Laboratory Standards Institute, Wayne, Pensilvania, EE.UU.

Clinical Laboratory Standards Institute (CLSI). 2013. Approved Standard Supplement Vet01-S2. Performance Standards for Antimicrobial Disk and Dilution Susceptibility Tests for Bacteria isolated from Animals. Second Informational Supplement. Clinical Laboratory Standards Institute, Wayne, Pensilvania, EE.UU.

Clinical Laboratory Standards Institute (CLSI). 2016. M100S 26th Edition: Performance Standards for Antimicrobial Susceptibility Testing. Clinical Laboratory Standards Institute, Wayne, Pensilvania, EE.UU.

da Silva AP, Schmith C, de Vargas A, Maboni, G, Rampelotto C, Schwab M, Escobar T, do Amaral A. 2014. Susceptibilidade antimicrobiana de Staphylococus spp. isolados de caes com pioderma superficial. Pesquisa Veterinaria Brasileira. 34(4):365-361. doi.org/10.1590/S0100736X2014000400010. 
Descristóphoris P, Fasola A, Benagli C, Tonolla M, Petrini O. 2011. Identification of Staphylococcus intermedius group by MALDI-TOF MS. Systematic Apply Microbiology. 34:45-51. doi: 10.1016/j.syapm.2010.11.004.

Descloux S, Rossano A, Perredten V. 2008. Characterization of new staphylococcal cassette chromosome mec (SCC$\mathrm{mec}$ ) and topoisomerases genes in fluoroquinolone- and methicillin- resistant Staphylococcus pseudintermedius. Journal of Clinical Microbiology. 46(5)1818-23. doi: 10.1128/ JCM.02255-07.

Devriese LA, Vancanneyt M, Baele M. 2005. Staphylococcus pseudintermedius sp. nov., a coagulase-positive species from animals. International Journal of Systematic and Evolutionary Microbiology. 55:1569-73. http://hdl.handle. net/1854/LU-328337.

Faires MC, Aucoin D, Weese JS. 2009. Inducible clindamycin-resistance in methicillin-resistant Staphylococcus aureus and methicillin-resistant Staphylococcus pseudintermedius isolates from dogs and cats. Veterinary Microbiology. 139:419-20.13. doi: 10.1016/j.vetmic.2009.06.016.

Gold RM, Lawon SD. 2013. Incidence of inducible clindamycin resistance in Staphylococcus pseudintermedius from dogs. Journal of Clinical Microbiology 51(12):4196-9. doi: 10.1128/JCM.02251-13.

Griffeth GC, Morris DO, Abraham JL, Shoper FS, Rankin SC. 2008. Screening for skin carriage of methicillin resistant coagulase positive and Staphylococcus schleiferi in dogs with healthy and inflamed skin. Veterinary Dermatology. 19(3):142-9. doi: 10.1111/j.1365-3164.2008.00663.x

Guardabassi L, Damborg P, Stamm I, Kopp PA, Broens EM, Toutain PL. 2017. Diagnostic microbiology in veterinary dermatology: present and future. Veterinary Dermatology. 46-e30. doi: 10.1111/vde.12414

Hnot M, Cole LK, Lorch G, Papich M, Rajala Schultz PJ, Daniels JB. 2015. Evaluation of canine minocycline and doxycycline susceptibility break points for methicillin resistant Staphylococcus pseudointermedius isolates from dogs. Veterinary Dermatology. 26:334-e71. doi: 10.1111/vde.12227.

Moodley A, Damborg P, Saxmose Nielsen, S. 2014. Antimicrobial resistance in methicillin susceptible and methicillin resistant Staphylococcus pseudintermedius of canine origin: Literature review from 1980 to 2013. Veterinary Microbiology. 171:337-41 doi: 10.1016/j.vetmic.2014.02.008.

OIE (Organización Mundial de Sanidad Animal). Código sanitario para los animales terrestres. Título 6. Capítulo 9. [ONLINE]. Disponible en: http://www.oie.int/es/normasinternacionales/codigo-terrestre/acceso-en-linea/. [Consultado: 09/06/2017].

Riley MC, Perreton V, Bemis DA, Kania S. 2016. Complete genome sequences of three important methicillin resistant clinical isolated of Staphylococcus pseudintermedius. Genome announcements 4 (5): e01194-16. doi: 10.1128/ genomeA.01194-16

Sasaki T, Kikuchi K, Tanaka Y, Takahashi N, Kamata S, Hiramatsu K. 2007. Reclassification of phenotypically identified Staphylococcus intermedius strains. Journal of Clinical Microbiology. 45(9):2770-8. doi: 10.1128/JCM.00360-07

Schissler JR, Hiller A, Daniels JB, Cole LK, Gebreyes WA. 2009. Evaluation of Clinical Laboratory Standars Institute interpretative criteria for methicillin resistance Staphilococcus pseudintermedius isolated from dogs. Journal of Veterinary Diagnostic Investigation. 21:684-8.
Steward CD, Raney PM, Morrell AK, Williams PP, McDougal LK, Jevitt L, McGowan JE Jr, Tenover FC. 2005. Testing for induction of clindamycin resistance in erythromycin-resistant isolates of Staphylococcus aureus. Journal of Clinical Microbiology. 43:1716:21-7. doi: 10.1128/JCM.43.4.17161721.2005

Vigo G, Giacoboni G, Gagetti P, Pasterán F, Corso A. 2015. Resistencia antimicrobiana y epidemiología molecular de cepas de Staphylococcus pseudintermedius aislados de muestras clínicas de caninos. Revista Argentina de Microbiología. 47(3):206-11.

Weese JS, van Duijkeren E. 2010. Methicillin-resistant Staphylococcus aureus and Staphylococcus pseudintermedius in veterinary medicine. Veterinary Microbiology. 140:418-29. doi: 10.1016/j.vetmic.2009.01.039.

Younis W, Mohammad H, Hostetler M, López-Péwrez D, Steussy C, Lipton M, Stauffcher C, Sullan S, Abd Al-Azeen MW, Husseim AA, Seteen N. 2017. Class II HMG-CoA reductase inhibitors targeting methicillin resistant Staphylococcus pseudintermedius. Journal of Advanced Veterinary Research 7(1):1-7. 